\title{
Führungswechsel bei DVS und GSI
}

Bedeutende personelle Veränderungen haben sich an der Spitze des DVS - Deutscher Verband für Schweißen und verwandte Verfahren e. V. sowie in der Leitung seiner Beteiligungsgesellschaft GSI - Gesellschaft für Schweißtechnik International $\mathrm{mbH}$ ergeben.

$\mathrm{M}$ it Wirkung zum 14. Februar 2013 hat Professor Dr.-Ing. Steffen Keitel, seit 2005 alleiniger Geschäftsführer der GSI, die Leitung der Gesellschaft auf eigenen Wunsch abgegeben, um sich zukünftig verstärkt der Leitung der Schweißtechnischen Lehrund Versuchsanstalt SLV Halle GmbH widmen zu können. Für die SLV Halle ist Professor Keitel bereits seit 1987 tätig - seit 1993 in der Geschäftsführung dieser Beteiligungsgesellschaft des DVS.

Zum neuen Geschäftsführer der GSI wurde vom Gesellschafterausschuss der GSI Dr.-Ing. Klaus Middeldorf berufen, der in diesem Zusammenhang zum 11. Februar 2013 seine Tätigkeit als Hauptgeschäftsführer des DVS beendete. Zu seinem Nachfolger an der Spitze des Verbandes hat das DVS-Präsidium den bisherigen Stellvertreter des Hauptgeschäftsführers Berthold Kösters berufen.
Sein Vorgänger Dr.-Ing. Klaus Middeldorf begann seine Tätigkeit beim DVS im August 1999 als Geschäftsführer der Forschungsver e i n i g u n Schweißen und verwandte Verfahren e. V. des DVS. Diese Aufgabe führte er auch

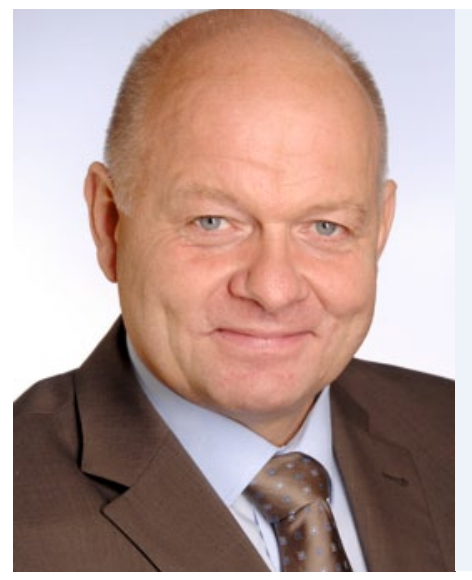

Berthold Kösters, neuer Hauptgeschäftsführer des DVS - Deutscher Verband für Schweißen und verwandte Verfahren e. V. weiter, als er zum 1. Januar 2006 mit der Hauptgeschäftsführung des DVS betraut wurde. „Der DVS, die GSI und auch die DVS Media GmbH arbeiten seit jeher eng zusammen“, erklärt Middeldorf. „Mit meinem Wechsel in die Geschäftsführung der GSI sind für mich neue persönliche Herausforderungen verbunden, die bisherige gute Zusammenarbeit zwischen dem Verband und seinen Beteili- gungsgesellschaften wird davon jedoch auch profitieren und neue strategische Möglichkeiten eröffnen.“

Berthold Kösters ist bereits seit 1992 in verschiedenen Funktionen beim DVS tätig. Als Stellvertretender Hauptgeschäftsführer war er seit 1. Januar 2006 vor allem für die Bereiche Finanzen, Personal und Organisation verantwortlich.

\section{BASF und Markor planen Joint Ventures in China}

$B^{\text {si }}$ ASF und Xinjiang Markor Chemical Industry Co., Ltd. (Markor), planen die Gründung von zwei Joint Ventures für die Herstellung von Butandiol (BDO) sowie Polytetrahydrofuran (Polytetramethylenätherglykol) in Korla, der im Nordwesten Chinas gelegenen autonomen uigurischen Region Xinjiang. Die Verträge der Gemeinschaftsunternehmen sind bereits unter Vorbehalt unterzeichnet und bedürfen noch behördlicher Genehmigungen.

Die Gemeinschaftsunternehmen beabsichtigen den Bau einer Anlage zur Herstellung von BDO sowie einer Anlage für Polytetrahydrofuran, die beide im Jahr 2015 in Betrieb gehen sollen. Die
Produktionskapazitäten der Anlagen in Korla sollen bei 100.000 Jahrestonnen BDO und 50.000 Jahrestonnen Polytetrahydrofuran liegen.

Polytetrahydrofuran wird hauptsächlich zur Herstellung elastischer Spandex-Fasern für verschiedenste Textilien eingesetzt, dient aber auch als chemischer Baustein für thermoplastische Polyurethane (TPU). Weitere Anwendungen sind thermoplastische Polyetherester, Polyetheramide und Gießelastomere. BDO findet bei der Herstellung von technischen Kunststoffen, Polyurethanen, Lösemitteln, Elektronikchemikalien und elastischen Fasern Verwendung.

\section{Loop erweitert Geschäftsführung}

Z ur Vorbereitung eines zu einem späteren Zeitpunkt geplanten Führungswechsels hat die Loop $\mathrm{GmbH}$ Marc Zick, Sohn des Gründers HansJürgen Zick, zum zweiten Geschäftsführer bestellt.

Damit soll ein reibungsloser Generationswechsel gewährleistet werden. Marc Zick übernahm bereits in 2008 die Leitung des Kunden-Service-Technikums. Das Unternehmen verfolgt auch weiterhin das Ziel, sich immer komplexeren Aufgaben im Bereich der Lohnfertigung chemisch-technischer Produkte zu stellen. 